\title{
From Knowing to Remembering: the Semantic-Episodic distinction
}

\author{
Louis Renoult ${ }^{1}$, Muireann Irish², Morris Moscovitch ${ }^{3}$, Michael D. Rugg ${ }^{1,4}$ \\ ${ }^{1}$ School of Psychology, University of East Anglia, Norwich, Norfolk, UK \\ 2 The University of Sydney, School of Psychology and Brain \& Mind Centre, Sydney, \\ Australia \\ ${ }^{3}$ Rotman Research Institute, Baycrest, Toronto, Ontario, Canada \\ ${ }^{4}$ Center for Vital Longevity and School of Behavioral and Brain Sciences, University of \\ Texas, Dallas, USA \\ Correspondence: I.renoult@uea.ac.uk
}

Keywords: Episodic Memory, Semantic Memory, Conceptual Knowledge, Reinstatement, Hippocampus, Anterior Temporal Lobe

\begin{abstract}
The distinction between episodic and semantic memory was proposed in 1972 by Endel Tulving and is still of central importance in Cognitive Neuroscience today. Data obtained in the last 30 years or so, however, support the idea that the frontiers between perception and knowledge and between episodic and semantic memory are not as clear cut as previously thought, prompting a rethinking of the episodic-semantic distinction. Here, we review recent research on episodic and semantic memory, highlighting similarities between the two systems. Taken together, current behavioral, neuropsychological and neuroimaging data are compatible with the idea that episodic and semantic memory are inextricably intertwined, yet retain a measure of distinctiveness, despite the fact that their neural correlates demonstrate considerable overlap.
\end{abstract}




\section{Glossary}

Episodic Memory: Recollection of personally experienced events situated within a unique spatial and temporal context (e.g., I remember reading "1984" at Hyde Park yesterday).

Familiarity: A collection of memory signals that support recognition memory in the absence of recollection (see Box 1).

Gist: Knowledge about a specific event or episode that encompasses its overall structure or central elements but without additional contextual, typically perceptual, details (e.g., what in general transpired at a particular birthday party [1]).

Recollection: Retrieval of consciously accessible, qualitative information about a past episode (see episodic memory above).

Remote Memory: Memories corresponding to events in the relatively distant past, usually a year or more ago. Though no definite passage of time defines remoteness, such memories typically date from relatively early in an individual's life, for example, childhood in the case of a young adult, or young adulthood in the case of a middle-aged or older individual. The term is descriptive and is neutral as to whether a particular memory is supported by episodic or semantic memory information or processes. Importantly, a remote memory needs not depend on access to the memory trace encoded concurrently with the event it represents; 
rather, it could reflect the content of a more recently encoded ('re-encoded') trace dating from the last time the memory was retrieved.

Schema: A large-scale knowledge structure that is extracted over multiple experiences. Whereas gist refers to a memory for the central elements of a specific episode (e.g. a recent birthday party), a schema captures similarities across multiple episodes or memories (e.g., what happens at birthday parties in general). Schemas are dynamic structures that evolve with new experiences, can influence how events are experienced, and how memories are encoded, retrieved and evaluated [see 2 for a fuller discussion].

Semantic Memory: General (encyclopaedic) knowledge as well as schematic representations of events distilled from lifelong experiences, retrieved independently from their original spatial or temporal context (e.g., "1984" was written by George Orwell; the definition of a birthday party, or knowledge of events that typically happen during birthday parties) 


\section{The episodic-semantic distinction in contemporary cognitive neuroscience}

Endel Tulving was the first to formally describe the distinction between episodic and semantic memory (see Glossary) in a book chapter published in 1972 [3], building on earlier philosophical [e.g., 4, 5, 6] and psychological [7-9] writings. The episodic-semantic distinction remained central to Tulving's thinking over subsequent years while undergoing significant evolution [e.g., 10, 11-14], and the distinction retains its importance in contemporary cognitive neuroscience. With a few notable exceptions, however, research on the cognitive neuroscience of episodic and semantic memory has formed largely separate research traditions. Nonetheless, findings stemming from these two traditions show important points of convergence, and arguably have reached a stage where a synthesis might be possible. We provide a selective review of these findings and discuss the extent to which they support Tulving's original notion that episodic and semantic memory are distinct (albeit highly interactive) memory systems. Almost 50 years since Tulving's original treatise, we reconsider the episodic-semantic distinction from the perspective of the modern era of cognitive neuroscience. Rather than emphasizing differences between these two forms of memory, we highlight their similarities at the cognitive and neural levels, focusing on the multifaceted ways in which they overlap and interact to support an array of sophisticated cognitive abilities.

\section{Semantic memory - neural underpinnings}

Semantic memory refers to our repository of general world knowledge, and the term is often used interchangeably with that of conceptual knowledge. The semantic knowledge base is typically viewed as including general (encyclopaedic) knowledge as well as schematic 
representations of events distilled from lifelong experiences, but that are retrieved independently from their original spatial or temporal context (e.g., "1984" was written by George Orwell; the definition of a birthday party) as well as personally-relevant conceptual knowledge (i.e., personal semantics, e.g., "I have always been quite shy"). The neural underpinnings of semantic memory have been the topic of several recent reviews [15-17] and are discussed here in abbreviated form. According to the highly influential sensorimotor ('embodiment') framework [e.g., 18, 19, 20 for early precursors, and, 21 for an opposing viewpoint], a concrete concept is encoded as a specific combination of sensory, motor and other modality-specific features, and is represented in the distributed pattern of neural activity that emerges when the neural elements representing these features are co-activated. Based partly on findings that neuropathology can result in seemingly category-selective impairments of conceptual knowledge [ 22 for discussion and critique], early proponents of the sensorimotor framework [e.g., 23, 24] argued that knowledge about different classes of objects can be modality-dependent. For example, whereas fruits are typically individuated by virtue of their constituent sensory features (e.g. shape, taste and color) rather than the actions that are associated with them (most fruits are eaten, for example), tools can be individuated by their associated actions (e.g., twisting, in the case of a screwdriver, vs. hitting, in the case of a hammer).

A sizeable body of functional neuroimaging evidence lends support to the idea that conceptual representations are distributed across modality-specific cortical regions. For example, in a study in which participants had to generate appropriate actions or color names in response to words denoting different objects, generating colors was associated with enhanced neural activity adjacent to color-sensitive cortical regions, whereas the opposite 
contrast identified enhanced activity in posterior temporal regions linked to action planning and motion processing [25] [for review, see 19]. More generally, it has been argued that the neural activity elicited when a concrete concept is brought to mind overlaps the activity elicited by perception of, and interaction with, an exemplar of the concept [26], leading to a blurring of the boundary between perception and knowledge. That is, to bring the exemplar to mind is to reinstate ['simulate' 18] the patterns of neural activity that would exist were the exemplar actually present in the environment [sometimes with an "anterior shift", such that the activity linked with retrieval of conceptual knowledge is localized slightly anterior to the activity elicited during perception, 27] [see also 26, 28]. This idea finds a strong parallel in theoretical ideas about episodic memory retrieval (see Episodic memory - neural underpinnings below). Furthermore, as implied by the example given above, the set of features activated by a given concept is not invariant, but rather can differ according to task and contextual factors. Selection of the features most appropriate for a given behavioral goal, and the resolution of interference between potentially competing feature sets, depends on control processes supported by a brain network centered on left inferior prefrontal cortex [e.g., 29, 30].

It is widely held that conceptual representations limited to the sensorimotor level are insufficient to support the full gamut of human conceptual processing and need to be combined in complex ways to create multi- or supra-modal representations. Notably, it is not obvious how a sensorimotor level of representation alone could support abstraction (e.g., the ability to categorize both roller skates and helicopters as modes of transport) or the representation of abstract concepts such as 'pious' or 'liberty' [for recent discussion of this issue see 31 and other papers in the same issue]. These and related considerations have long 
motivated proposals for a mechanism that allows not only for the integration of sensorimotor information arising from different modalities in real-time, but for the encoding of covariances in this information that emerge over time. Such a mechanism could support the formation of higher-order representations that are abstracted away from modality-specific information [e.g., 32]. According to one prominent model [33], the mechanism takes the form of a computationally homogeneous 'hub' that receives input from activated sensorimotor feature sets and forms representations based on patterns of covariance across the different feature sets [this usage of 'hub'- which encompasses specific integrative, computational and representational functions - differs from its typical usage in network neuroscience, when no specific computational role is implied, 34]. Largely on the basis of neuropathological evidence from the syndrome of semantic dementia (see Episodic and Semantic Memory in Neurodegenerative disorders below), along with supporting evidence from transcranial magnetic stimulation and $\mathrm{fMRI}$, the proponents of this 'hub and spoke' model have argued that the hub is localized to bilateral anterior temporal cortex, centered on the anterior third or so of the fusiform gyrus [15]. This proposal has not gone unchallenged, with others arguing that amodal conceptual representations depend critically on more posterior regions of temporal cortex [35, 36], or on multiple, heteromodal cortical 'convergence zones' [37, 38]. Of particular relevance here, it has been proposed that a 'general semantic network' (Figure 1A) comprising, in addition to anterior temporal cortex, many of the same regions that are held to comprise the 'core recollection network' (see Glossary and Episodic memory - neural underpinnings below), operates as a distributed convergence zone to support conceptual representations at varying levels of abstraction [38]. Consistent with its heteromodal characterization, neural activity in the regions belonging to this network covaries with amount of conceptual processing across a wide range of experimental tasks, materials and 
manipulations [including lexicality (word vs. pseudo-word), word frequency, word concreteness, and number of thematic associations; see [38] for review]. Furthermore, multivoxel pattern analysis (MVPA of fMRI data) has revealed that the patterns of activity within these regions can support decoding of semantic/conceptual content [39].

In summary, there is some consensus that conceptual (semantic) knowledge depends upon a combination of neural activity distributed across cortical regions that support modalityspecific information, and neural processing within heteromodal cortical regions that operate on these patterns of modality-dependent activity to create representations in rich, highdimensional feature spaces.

\section{Episodic memory - neural underpinnings}

Episodic Memory refers both to a hypothetical 'episodic memory system', which encodes, stores, and allows access to 'episodic memories', and also to the memories themselves, which are often held to have unique phenomenological attributes. The distinction between these two usages is usually signaled only by the context in which they are used. Episodic memory in the latter sense refers to recollection of personally experienced events situated within a unique spatial and temporal context (e.g., I remember reading "1984" at Hyde Park yesterday). In Tulving's original conception [11], episodic memory is tightly associated with awareness of the self situated in subjective time (autonoetic awareness), which permits events to be re-experienced (episodic retrieval) or 'pre-experienced' (episodic future thought) (see Box 3).

According to a long-standing framework [see 40 for review], episodic memories are a byproduct of the processing engaged by an event as it is experienced. As the event unfolds, 
some of the cortical activity it elicits is encoded in the hippocampus as a content-addressable memory in which the patterns of cortical activity that represent the features are bound into a memory representation. Retrieval of an episodic memory (recollection; see Glossary) occurs when a retrieval cue activates a stored hippocampal representation sufficiently to cause 'pattern completion', restoring the representation to an active state. In turn, this leads to reactivation of the encoded pattern of cortical activity, and hence access to mnemonic content [see 40 for discussion of the preconditions for successful episodic retrieval].

Findings from functional neuroimaging studies provide compelling support for this general framework. Relative to unsuccessful recollection, successful recollection is associated not only with hippocampal activation, but also with reinstatement of some of the cortical activity that was elicited when the recollected event was initially experienced. Retrieval-related 'reinstatement effects' were first identified through univariate analyses of Positron Emission Tomography (PET) and, subsequently, fMRI BOLD signal changes $[40,41]$, when they take the form of overlap between regions demonstrating differential activity between two or more study conditions, and regions demonstrating study condition-dependent differences in recollection-related activity (see Fig. 2 for an example). Retrieval-related reinstatement has also been demonstrated with MVPA of fMRI data. Such findings have been reported both for linear classifiers trained on study data and used to classify corresponding test trials [e.g., 42], and for analyses of the similarity of patterns of activity elicited during the encoding and subsequent retrieval of specific episodes [e.g., 43].

As is illustrated in Fig. 1B, recollection-related enhancement of BOLD activity is found not only in content-sensitive cortical regions but also in regions that, along with the hippocampus, have been proposed to comprise a general or 'core' recollection network [44, a very similar, 
if not identical, network is active during autobiographical memory retrieval and 'episodic future thought', 45]. The network was so named because it is engaged during successful recollection seemingly regardless of the nature of the recollected content or the memory test used to elicit recollection. The cortical components of the network include parahippocampal, medial prefrontal (MPFC), and posterior cingulate/retrosplenial cortex, along with left angular gyrus and left middle temporal gyrus. Importantly, while activity within the network is enhanced in a generic manner when recollection is successful, studies employing MVPA have reported that retrieved content can be 'decoded' from most, if not all of its members $[43,46]$. These findings indicate that the sensitivity of the network to successful recollection goes beyond a simple elevation of global activity, and that retrieval-related reinstatement is not confined to cortical regions - for example, 'the 'fusiform face area' or auditory cortex (Fig. 2) - that are specialized for information belonging to a specific modality or perceptual category.

The network depicted in figure 1B represents the outcome of a contrast between retrieval cues that elicited recollection- versus familiarity-driven (see Glossary and Box 1) recognition memory judgments. Whereas such contrasts allow the neural correlates of successful recollection to be identified while holding stimulus and task factors constant, they cannot identify neural regions that are sensitive to the cognitive demands of episodic retrieval independently of the outcome of the retrieval attempt. Consistent with the notion that the left inferior prefrontal cortex supports semantic control processes, however (see previous section), it has been reported that the region is more active during episodic memory tests that place high, as opposed to low, demands on the processing of retrieval cues [e.g. tests of source memory vs. simple item recognition 47, 48]. Additionally, it has been reported that members of the core recollection network not only demonstrate enhanced activity during 
successful recollection, but also enhanced functional connectivity with parietal and dorsolateral/dorsomedial frontal regions comprising the 'frontoparietal' and 'cinguloopercular' control networks [49, see also 50]. Regions belonging to these networks are held to act as 'flexible hubs' that couple with different functional networks depending on task demands [51]. Thus, enhanced functional connectivity with the core recollection network likely reflects the engagement of domain-general control processes in support of postretrieval operations such as selection of an appropriate behavioral response on the basis of recollected content. Perhaps not uncoincidentally (see next section), in one study [52] increased demands on semantic processing were reported to result in enhanced functional connectivity between members of the general semantic network and networks implicated in domain-general cognitive control.

\section{Episodic and semantic memory retrieval - overlapping neural correlates}

As is evident from Figure 1, the core recollection network overlaps strongly with the general semantic network. Specifically, the two networks share essentially the same parahippocampal, middle temporal, ventral parietal and midline frontal and posterior regions. Conspicuous by its absence in both Figures $1 \mathrm{~A}$ and $1 \mathrm{~B}$ is ventrolateral anterior temporal cortex, the putative 'semantic hub' that plays a central role in the 'hub and spoke' model of semantic cognition described in the section Semantic memory - neural underpinnings above. As has been noted previously [e.g., 53, 54], the seeming absence of the ATL from the semantic network likely reflects the degradation of signal quality in the region caused by magnetic susceptibility artefact in the vicinity of the sphenoid sinus. Consistent with this possibility, $\mathrm{fMRI}$ studies employing sequences optimized to detect BOLD signals from 
ATL have reported that its activity, especially in anterior fusiform gyrus, is enhanced during conceptual processing $[54,55]$. Although it has yet to be established whether the ATL consistently demonstrates episodic retrieval effects with optimized fMRI, figure 1C, which illustrates findings from a single large-scale study, suggests that this is a distinct possibility.

Also evident in Figure 1 is the presence of the hippocampus in the core recollection network but not in its semantic counterpart. The greater prominence of the hippocampus in the recollection network is perhaps unsurprising in light of the key role of this structure in both the encoding and reinstatement of the patterns of cortical activity set in train by episodes as they are experienced (see previous section). Indeed, the hippocampus may act as a hub that supports integration of information distributed across the recollection network, and helps distinguish it from the semantic one, despite the substantial of overlap between the two networks. It should be noted, however, that although the hippocampus might ordinarily play only a limited role in the retrieval of conceptual information [see next section, but see also Box 4 and 56, 57 for evidence that the hippocampus supports performance on certain semantic fluency tasks], it likely does play a role in its acquisition, at least for some kinds of information. This role is evident in numerous studies that report a marked impairment in the ability of patients with hippocampal damage to acquire new factual (encyclopedic) knowledge, even after intensive, repeated training $[58,59$, see 60 for discussion of possible exceptions to these findings in cases of hippocampal damage in early childhood, and see 61, 62 for discussion of the possibility of fast mapping in adults]. Additional evidence possibly implicating the hippocampus in semantic cognition comes from single unit studies of the human hippocampus. These studies identified cells that respond to specific individuals, places or objects (and to stimuli associated to these concepts) whether the entity is presented as an 
image, or a written or spoken word [reviewed in 63,64]. Dubbed "concept cells", they have been interpreted as coding semantic representations that "constitute the building blocks for declarative memory functions" [63; page 592]. One has to note, however, that these responses to specific individuals, places or objects may only represent one form (and perhaps not the canonical form) of semantic memory. Semantics frequently requires extraction of statistical regularities over different exemplars of a category [e.g., we develop a representation of the concepts of cats through learning about many different cats, typically not through a single individual, but see 65 for a recent example of an exemplar-memory model of category learning]. An alternative, though not mutually exclusive, interpretation is that concept cells are experiential, episodic cells that link a specific entity to other aspects of an experienced event involving the entity $[66,67]$.

What underlies the overlap between the semantic and recollection networks? We propose that the answer lies in the fact that the content of an episodic memory typically comprises a conjunction of familiar concepts and episode-specific information (such as sensory and spatial context), much as the episodic interpretation of concept cells suggests. Thus, recollection of a prior episode entails the reinstatement not only of contextual information unique to the episode, but also of the conceptual processing that was engaged when the recollected event was experienced [see also 68]. From this perspective, 'recollection success effects' in cortical members of the core recollection network do not reflect processing that supports episodic memory per se, but rather, the reinstatement of the conceptual processing that invariably underpins our interactions with the world in real-time [e.g., 12, 69, 70]. The importance of such processing for episodic memory is well-illustrated by the syndrome of semantic dementia (see section Episodic and Semantic Memory in Neurodegenerative disorders), when recollection of meaningful information is selectively impaired [e.g., 71, 72]. Of importance, if 
the foregoing proposal [a similar proposal was advanced in 70] is correct then, from the standpoint of episodic memory, regions belonging to the core recollection network deserve no more privileged a position theoretically than any other cortical region in which retrievalrelated reinstatement can be identified.

We note that the foregoing proposal leaves open the important question of the specific roles played by these different regions in semantic (and, by implication, episodic) memory [see 73 for recent discussion of this question]. This question is far from resolved, although numerous and sometimes conflicting proposals have been advanced. Interestingly, several brain regions have been proposed to serve as an interface between semantic and episodic memory, such as the parahippocampal cortex and the posterior cingulate cortex [16] or the perirhinal cortex [74]. As to parahippocampal cortex, it has variously been proposed that it may play a role in semantic associative processing [75-77], while others have discussed its importance in processing scenes $[66,78]$ and objects that evoke an awareness of surrounding space [79]. In the case of the angular gyrus, proposals have ranged from the idea that the region contributes to combinatorial processes [on-line construction of concepts such as 'fast truck', or 'plaid jacket' etc. 80,81 ] to the proposal that it does not play a specialized role in semantic cognition at all, but instead supports a domain-general 'buffer' [82]. Similarly diverse proposals have been put forward regarding the roles of other members of core network in semantic cognition. In the case of the middle temporal gyrus, for example, proposals range from a role in the conceptual representation of concrete objects[73] to semantic control [83]. And ventral mPFC has been proposed to support processing of the affective significance of concepts [16], to be a critical brain region for the encoding and storing of cognitive schemas (see Glossary) 
supporting the extraction of commonalities between events $[2,84-86]$, and to play a role in memory consolidation $[87,88]$.

Although the proposal that recollection success effects in the core network reflect the reinstatement of conceptual processing is both parsimonious and, we contend, consistent with the available evidence, it lacks direct support. fMRI studies examining the neural correlates of successful recollection have invariably employed meaningful experimental items such as concrete words or pictures of objects and have typically done so in the context of study tasks that require or encourage semantic elaboration. To our knowledge, with the exception of [89], there are no published studies in which recollection effects were contrasted according to the amount of semantic or conceptual processing engaged during encoding [although see 90 for a study in which encoding was manipulated but the subsequent memory test did not allow identification of items recognized on the basis of recollection rather than familiarity]. In [89], the memory test required a discrimination between unstudied items and items subjected to semantic or non-semantic study. Retrieval effects in the core network were not fully explored, but intriguingly, one member of the network (left parahippocampal cortex) was reported to demonstrate a greater recollection effect (operationalized as greater activity for correct than incorrect source judgments) for semantically than non-semantically studied items. This finding is consistent with the present proposal, but it remains to be established whether, as predicted by the proposal, recollection-related activity within the core network as a whole co-varies with the amount of semantic processing accorded a recollected episode when it was first experienced. 


\section{Evolution and transformation of episodic and semantic memories over time}

Thus far, we have discussed episodic and semantic memories without reference to the possibility that their content and neural underpinnings might vary over time. There is, however, a long-standing literature documenting that memory representations can be highly dynamic, shifting their dependence from the hippocampus and adjacent regions of the medial temporal lobe (MTL) to other neocortical regions - a phenomenon often referred to as systems consolidation [66, 67, 91-93]. In recent years, systems consolidation has become increasingly intertwined with the construct of memory 'semanticization' and schematization, processes by which semantic knowledge and schemas [2] emerge from episodic memory or assimilate aspects of it.

Early studies and theories of memory consolidation, beginning with Ribot and reiterated for almost a century, typically did not distinguish between episodic and semantic memory [67, 94-96]. Among the first to realize the importance of the episodic-semantic distinction for theories of memory consolidation were Kinsbourne and Wood [97]. They proposed that traumatic amnesia affected only episodic memory, regardless of the age of the memory, and left semantic and schematic memory relatively preserved. Cases in which remote episodic memories appeared to be preserved were attributed to semanticization or schematization through repeated re-encoding (see Glossary: Remote memory), allowing them to achieve the status of personal facts $[98,99]$.

In an important development of the 'standard' model of consolidation, McClelland et al. proposed that the hippocampus maintains episodic representations of an event while communicating with ('instructing') the neocortical system to incorporate information about the event into its knowledge structure [100]. It was argued that, in order to protect the 
cortical network from catastrophic interference, learning had to be slow, thus providing a principled explanation for the extended time period that systems consolidation was assumed to take. Of importance, the model proposes that in the process of incorporating an episodic memory into a semantic network, the episodic component, initially dependent on the hippocampus, is lost. This represents an important point of divergence from the standard model, in which episodic information is retained in the neocortex along with semantic information (see below).

Incorporating the original idea of Kinsbourne and Wood [97] and the complementary learning perspective [100], 'multiple trace theory' [MTT; 101] proposed that the hippocampus supports episodic memories for as long as they exist. By contrast, the theory proposed that semantic memories depend upon the neocortex, which extracts statistical regularities across distinct episodes. Thus, hippocampal damage should have a profound effect on retention and retrieval of episodic memories of any vintage, while leaving semanticized and schematized memories relatively intact.

While receiving empirical support $[66,102]$ [see also $67,103,104$ for examples of convergent findings from studies of experimental animals], MTT has also been subjected to several critiques [e.g., 93, 105, 106-108]. The essence of the theory, however, resonates with the recurring theme of the present review that episodic and semantic memory are intertwined, yet retain a measure of functional and neural distinctiveness. Since its inception, MTT has been extended $[67,104,109]$ to propose that episodic memories can become transformed to more semantic or schematic versions with time and experience [see section Episodic and Semantic Memory in Neurodegenerative disorders]; indeed, in some cases, both the original and the semanticized or schematic version of a memory co-exist and engage in dynamic 
interaction with one another. According to this Trace Transformation Theory, the specific neocortical regions supporting transformed memories differ depending on the kind of information that is retained and retrieved. Correspondingly, for complex events the transformed memories might depend either on event schemas, or on the gist of the event [see Glossary; 1, 110-112]. Increased activation of ventral mPFC - believed to be implicated in processing schemas [2] - and decreased hippocampal activation have both been reported as details are lost and memories become more gist-like and schematic [1, 2, 102, 112], particularly for memories that are congruent with existing schemas $[113,114]$. Even when details of remote memories are retained, along with continuing hippocampal activation, there is increased vmPFC activation over time $[115,116]$. Which memory of an event (e.g., its semanticized or schematic version or the detailed episodic memory of the original event) predominates at retrieval will depend on a variety of factors, such as contextual factors and processing demands [see sections Semantic memory - neural underpinnings and Episodic memory - neural underpinnings above], in addition to the availability of one or the other type of information [see also 117]. Retrieval of complex memories thus depends on the coordinated activation of different combinations of regions ['Process Specific Assemblies', 66, $118,119]$ belonging to neural networks underlying episodic and semantic memory.

The neuroimaging evidence reviewed to date strongly suggests that successful recollection necessitates the reinstatement not only of sensory-perceptual contextual information characteristic of the original experience, but also the semantic representations and conceptual processing that occurred during that experience. Rather than viewing episodic and semantic memory as dichotomous or mutually exclusive entities, the marked neural overlap 
between these forms of memory suggests that we must move towards considering the dynamic interplay of sensory-perceptual and conceptual elements during reinstatement of a recollected experience. One way in which we can potentially test this proposal is to examine how progressive neural insult of key structures implicated in episodic and semantic memory impacts related putative functions, including event recollection and event construction. We next consider how studies of these processes in the dementias have provided important insights into the brain regions that not only are implicated in, but essential for, successful recollection.

\section{Episodic and Semantic Memory in Neurodegenerative disorders}

In recent years in particular, the study of neurodegenerative disorders has provided important insights into the neurocognitive architecture of the episodic and semantic memory systems, as well as their respective interactions. Alzheimer's disease (AD), characterized by relatively selective MTL degeneration in its early stages, is often invoked as a lesion model for episodic memory, given the prominent deficits in episodic memory for visual and verbal information that are characteristic of this condition [120, 121]. Early studies of autobiographical memory revealed negative temporal gradients in AD by which retrieval of recent, presumably episodic, memories was compromised in comparison with relatively preserved remote retrieval, assumed to reflect semanticized memory representations [122, 123]. Notably, however, more fine-grained assessment of autobiographical memory that focuses on episodic (e.g., 'internal') content $[124,125]$ has suggested the presence of deficits in episodic memory that extend across the lifespan in $A D$, manifesting in flat retrieval gradients. Moreover, examination of the 'external' content (commonly assumed to reflect 
semantic or non-episodic details) of autobiographical narratives reveals a confection of perceptual and conceptual information that ranges in specificity from well-defined episodes to decontextualized semantics [126]. This amalgam of episodic and semantic representations enmeshed within the autobiographical narrative resonates with continuum-based accounts of personal semantics [127] and suggests that episodic-semantic interdependencies are critical for autobiographical retrieval, irrespective of the age of the memory [128].

The fuzziness of the boundaries between the episodic and semantic memory systems (see Episodic and semantic memory retrieval-overlapping neural correlates above) is accentuated when considering converging evidence from semantic dementia (SD), a younger-onset neurodegenerative disorder typified by an amodal loss of conceptual knowledge associated with progressive degeneration typically starting in the anterior temporal lobes and spreading to posterior temporal and prefrontal regions. Although predominantly a disorder of semantic processing, SD has illuminated our understanding of complex cognitive processes traditionally labelled as 'episodic'. Despite profound semantic impairments, these patients present with relatively spared episodic memory for recent experiences and intact visuospatial processing, at least in early stages of the disease [129]. Studies of autobiographical memory in SD regularly document a step function whereby recent memories remain relatively intact in the context of impoverished remote recall $[124,130]$. This relative sparing of recent episodic memory in SD has been suggested to reflect intact perceptual and visuospatial processing, crucial for episodic memory likely mediated by relatively preserved posterior temporoparietal regions $[121,131]$, at least in early stages of the disease. The retrieval of recent experiences tends to rely more heavily on sensory-perceptual representations in healthy individuals [132, 133]. It is not surprising, therefore, that the recent event narratives of SD patients tend to be 
more heavily weighted towards the provision of perceptually rich contextual details, in conjunction with whatever residual conceptual information remains available, some of which may be unrelated to the main event being described [126]. In contrast, the well-documented remote memory impairments in SD mesh well with the observation of a shift towards more semanticized accounts, even in healthy adults, thus taxing the semantic processing system disproportionately [134].

\section{Episodic-semantic interactions during event construction}

The harnessing of intact sensory-perceptual contextual details to support recent episodic retrieval is of particular interest when considered in the context of episodic construction. Current theoretical frameworks hold that the capacity to construct imagined future events, termed episodic future thinking (see Box 3), hinges upon the extraction of contextual details from episodic memory, which are recombined in a flexible manner to construct new and novel event representations [135]. While episodic representations remain available and accessible to SD patients, these representations do not appear sufficient to support the construction of novel future events [136]. A consistent finding in the literature is of marked future thinking impairments in SD despite intact episodic memory for perceptual and sensory information $[137,138]$, with the majority of future events recapitulated from past experiences. These findings lend compelling support for the semantic scaffolding hypothesis $[117,139]$, which views semantic representations as imparting the essential organizational framework to guide (re)construction of the past, simulation of the future, and the realization of spatially coherent scenes (see Box 4). With the appropriate schematic framework in place, relevant sensoryperceptual details can be co-opted into the simulation to form a coherent mental 
representation. Note that this proposal is similar to the one made above (see Episodic and semantic memory retrieval - overlapping neural correlates), that recollection of a prior episode entails the reinstatement not only of contextual information unique to the episode, but also of the conceptual processing that was engaged when the recollected event was experienced. Interestingly, though, inspection of the 'non-episodic' content of such simulations in SD suggests an inflation of 'external' (seemingly non-episodic) details [140], a somewhat counterintuitive finding in a population characterized by stark semantic impairments [see Outstanding Questions]. Application of fine-grained scoring taxonomies however reveal that a large proportion of these external details comprise temporally extended episodes, which in turn are negatively associated with semantic processing capacity, and correlate with integrity of the left posterior hippocampus [141]. As such, with progressive deterioration of the conceptual knowledge base, SD patients default to a predominantly episodic narrative, mostly likely reflecting a compensatory strategy whereby content is salvaged from the least-compromised memory system. This pattern is also evident for the mental construction of static scenes with no temporal constraints (i.e., scene construction, see Box 4), with SD patients effectively repurposing previously experienced episodes in their entirety [142].

The asymmetrical impairments during future, relative to past, (re)construction in SD highlight the central role for conceptual representations in knowledge manipulation and generalization during the construction of new experiences. Rather than viewing complex constructive processing as the sole remit of episodic memory, contemporary theories of memory function must consider the intricate interdependencies between these representational systems. Irrespective of whether we remember the past or envisage the future, episodic and semantic 
elements are inextricably intertwined, with the relative weightings of these representations in the resultant construction hinging upon task demands and the integrity of the underlying memory system $[117,143]$. This proposal resonates with the prescient observations of Ingvar who posited that semantic memory is "mainly involved in the cognitive or future consequences and meaning of events" [144, p. 129]. Semantic memory thus provides the necessary organizational framework from which detailed events can be constructed. As such, interactions between the episodic and semantic memory systems, both being declarative (i.e., conscious, but see Box 2) and subject to control processes, provides us with optimal flexibility to complete an array of complex computations. Just as an episode can be described in terms of its gist, or its specific sensory-perceptual details, so too can we focus on different aspects of a semantic concept and relate it to higher- or lower-order concepts (e.g., a dog can be viewed as a mammal or a specific breed). This confers an impressive flexibility enabling us to dynamically shift between different representations at varying levels of specificity according to task demands, contextual factors, and integrity of the underlying memory systems [see also 117].

\section{Concluding remarks}

When Endel Tulving first proposed that a distinction be drawn between semantic and episodic memory he considered it to be a "pre-theoretical position" that did not imply they were necessarily functionally distinct in any deep sense [3]. Over time, he came to view the two forms of memory as being both functionally and neurally dissociable [10-13], while never failing to emphasize that normal cognition depends on their intimate interaction. Above, we reviewed diverse evidence that suggests that although episodic and semantic memory might 
represent the expression of different memory systems as Tulving proposed, the boundaries between them, whether anatomically or functionally defined, are not as distinct as Tulving's proposal may have led one to believe. Behavioral, neuropsychological and neuroimaging data converge to indicate that episodic and semantic memory are inextricably intertwined and that their neural correlates largely overlap. Yet current evidence also suggests that the relative weightings of episodic and semantic representations of the same event vary with time and with task demands, and that the neural correlates of episodic and semantic memory maintain a degree of distinctiveness. It will be of considerable interest to see whether future research leads to a clearer delineation of these boundaries accompanied by a better understanding of how the systems interact, or to the further dissolution of these boundaries and their replacement with a different perspective on Remembering and Knowing.

\section{Text Box 1: The concept of familiarity in episodic and semantic memory}

The essence of episodic memory lies in the process of recollection, by which one can not only reinstate the contextual details of an event but can mentally re-experience it. It is widely held however that in tests of recognition memory, judging whether an item is old or new can also be achieved by evaluating its 'familiarity', a sense of prior experience bereft of contextual details. Familiarity, however, is "not a well-grounded theoretical concept" [145]; it is defined by exclusion - recognition memory in the absence of recollection - and is likely based on multiple sources of information that can each support a judgment that a stimulus event has been recently experienced. A celebrated example of such information is the enhanced 'perceptual fluency' of recently studied objects and words that, under appropriate 
circumstances, can lead to a positive recognition memory judgment and a subjective sense of familiarity [146, 147]. More recently, it has been claimed that high levels of 'conceptual' fluency can likewise support familiarity-based recognition [e.g., 148, 149, see also 150, 151].

A popular behavioral method - the 'Remember/Know procedure - for segregating recognition memory judgments according to whether they are based on recollection or familiarity was proposed by Tulving [11] not with the aim of supporting 'dual-process' models of recognition memory, but rather, to separate memory judgments supported by episodic (associated with autonoetic consciousness or 'remembering') as opposed to semantic (associated with noetic consciousness - 'knowing') memory. Whereas relatively few of the many researchers who currently employ the Remember/Know procedure do so on the assumption that they are dissociating episodic and semantic memory (as noted above, familiarity is a catch-all term for a variety of memory signals), to the extent that conceptual processes play a role in familiaritybased recognition [148-152] 'know' judgments may indeed sometimes fulfill the role Tulving attributed to them.

As noted in the main text, the semantic network overlaps closely with the recollection network and 'recollection success effects' in the core recollection network may reflect reinstatement of conceptual processing. From this perspective, familiarity arising from conceptual fluency might involve very weak (and hence undetectable) reactivation effects in the core recollection network. Indirect support for this possibility comes from the finding that study content can be decoded from core recollection regions almost as accurately for "Know" as for "Remember" judgments, despite the absence of an effect on fMRI BOLD signal magnitude [46]. 


\section{Text Box 2: Episodic and Semantic Memory: both Implicit and Explicit?}

Recently, data have accumulated that suggest that simple views of dichotomies such as explicit/implicit or declarative/non-declarative may have to be revised, and that the presence or absence of conscious awareness may not always be the best way to categorize memory systems [153-156]. For instance, in the domain of episodic memory, it has become clear that episodic memories can implicitly influence performance on a variety of tasks [e.g., 157, 158, 159]. Indeed, Moscovitch and colleagues have proposed that episodic retrieval has two stages. The first stage is fast and non-conscious and involves an interaction between a retrieval cue and a memory representation [or "ecphory", 160] that makes episodic information available to on-going cognition, whereas a second, slower, stage is required for the retrieved information to be re-experienced $[66,159,161]$. The first stage involves the hippocampus, while the second depends on interactions between the hippocampus prefrontal and parietal cortex.

Turning to semantic memory, although it is typically referred to as explicit/declarative, the notion that it can be expressed either implicitly or explicitly is not new $[100,162,163]$. For example, there is evidence that meaningful stimuli such as words automatically activate their meanings so as to bias a subsequent behavioral judgment even under presentation conditions that seemingly preclude their conscious identification [reviewed in 164]. Moreover, brain regions thought to play a role in semantic processing, such as temporal and inferior parietal cortex, appear to be sensitive to semantic priming manipulations regardless of whether the prime was processed implicitly or explicitly [165-167, see also 168]. Taken together, recent evidence thus suggests that episodic and semantic memory may involve both implicit and explicit processes. 


\section{Text box 3: Mental time travel}

Relevant to the distinction between episodic and semantic memory is the unresolved question of temporality, or 'mental time travel' $[11,13,169,170]$. In his later formulations, Tulving placed increasing emphasis on the conjunction of self, agency, ownership and subjective temporality in promoting a sense of self-knowing or autonoetic consciousness [13, 171]. By this view, a defining feature of episodic memory is a first-person subjectivity involving the experience of mentally reliving the original event [172]. Semantic memory, by contrast, was associated with noetic (knowing) consciousness by which one would simply 'know' that events had transpired in the absence of a feeling of re-experiencing [172]. As noted elsewhere (Box 1), such phenomenological distinctions were argued by Tulving as amenable to systematic empirical study via the 'Remember/Know' procedure. Subsequently, a number of related procedures have been developed that permit rememberers to classify different subjective experiences accompanying memory retrieval [e.g., 173, 174-176].

As noted above, Tulving accorded episodic memory a unique status in conferring the phenomenological sense of mentally reliving the past. By this view, merely retrieving the conjunction of "what, where, and when" is not sufficient to qualify as an episodic memory, a proposal that elevated human episodic memory above that of the rudimentary "episodic-like" retrieval displayed by corvids and other non-human animals [177, but see 178$]$. A critical question, therefore, is whether the contemporary empirical focus on the products of episodic and semantic memory (i.e., their representational content) downplays the importance of phenomenally-based distinctions between the two types of memory. Moreover, if autonoetic experience is an inherent part of episodic retrieval, how do we reconcile this with current neurobiological models of memory which seemingly have no place for subjective experience? 
Some recent proposals dissociate memory traces from related subjective experiences [i.e., from autonoesis, 172] [or from the capacity to travel mentally in time, 179], acknowledging that there may be no clear dividing line between episodic and semantic memory traces in the brain, and leaving open the question of the neural bases of autonoesis and mental time travel [see Outstanding Questions]. Several brain regions have been proposed to play a role in mental time travel, including prefrontal [e.g., 180] and parietal cortex [e.g., 181], and the hippocampus [e.g., 178]. In the case of the hippocampus, its involvement in mental time travel would be consistent with its stronger association with episodic than with semantic retrieval [see Episodic and semantic memory retrieval - overlapping neural correlates]. Evidence implicating the hippocampus in mental time travel, however, remains inconclusive [182].

\section{Text box 4: Declarative Memory and Spatial Cognition}

The distinction between episodic and semantic memory has been applied to the domain of spatial cognition. In everyday situations, having a general conceptual outline of our spatial environment, as opposed to remembering specific instances of travelling somewhere, is often sufficient to navigate successfully from A to B. However, when visiting new places, taking a detour from a well-worn path, or attempting to remember a particular route one once took, a fine-grained representation more closely resembling contextually rich episodic retrieval is required [183]. The hippocampus is thought to be essential for representing spatial details at high resolution, providing the essential spatial context during episodic retrieval, but less so for the schematic or conceptual representation of space $[1,184,185]$. In line with this view, patients with hippocampal amnesia have been reported to show an impaired capacity to 
imagine coherent scenes in rich detail [such as lying on a beach on a sunny day; 186]. While the descriptions produced by amnesic patients contain the requisite semantic information, their overall constructions are spatially fragmented. Conversely, scene construction appears to be relatively well preserved in patients with semantic dementia, who despite marked deterioration of the conceptual knowledge base, can nevertheless imagine and describe scenes in rich detail, at least in early stages of the disease [142]. This pattern of preservation may reflect the relative sparing of the right hippocampus [see also 187], or integrity of posterior parietal brain structures such as the retrosplenial cortex [188]. Thus, while the hippocampus has been ascribed a central role in constructing the requisite spatial context across past, future, and hypothetical scenarios [189-192], it may be important to consider the role of other structures and laterality effects in this context.

Although preferentially responsive to the retrieval of episodic memories, mounting evidence suggests significant involvement of the hippocampus in semantic processing tasks, particularly those containing spatial information ("furniture in a living room") [e.g., 57, 193]. Further, amnesic patients with medial temporal lobe damage display selective deficits on semantic fluency tasks involving spatial as opposed to non-spatial categories [56]. Together, these findings can be interpreted in light of the idea that the hippocampus provides a domaingeneral form of scene construction or "spatial scaffolding"; for example, thinking about a conceptual category like "kitchen" often instantiates a related scene in the mind's eye. In a study that varied spatial content while comparing hippocampal activation during episodic and semantic memory tasks, researchers identified significant hippocampal activation for all instances of memory retrieval (episodic or semantic) in which a spatial context was evoked [194]. Moreover, spatial retrieval, irrespective of memory type, was associated with activation of posterior brain structures typically implicated in visuospatial processing. The 
authors interpreted their findings as evidence for the hippocampus supporting spatial, rather than relational, content during retrieval, and serving as a key interface between the episodic and semantic memory systems.

\section{References}

1. Robin, J. and Moscovitch, M. (2017) Details, gist and schema: hippocampal-neocortical interactions underlying recent and remote episodic and spatial memory. Current Opinion in Behavioral Sciences $17,114-123$.

2. Gilboa, A. and Marlatte, H. (2017) Neurobiology of Schemas and Schema-Mediated Memory. Trends in Cognitive Sciences 21 (8), 618-631.

3. Tulving, E. (1972) Episodic and Semantic Memory. In Organization of memory., pp. 381-403, Academic Press.

4. Bergson, H. (1911) Matter and memory, London: George Allen and Unwin.

5. Russell, B. (1921) The analysis of mind, George Allen \& Unwin, London.

6. Furlong, E.J. (1951) A Study in Memory, Thomas Nelson and Sons, London.

7. Claparede, M.E. (1911) Recognition et moiite. Archives de Psychologie (11), 79-90.

8. Reiff, R. and Scheerer, M. (1959) Memory and hypnotic age regression, New York, International Universities Press.

9. Bruner, J.S. (1969) Modalities of memory. In The Pathology of Memory (Talland, G.A. and Waugh, N.C. eds), pp. 253-259, New York: Academic Press.

10. Tulving, E. (1983) Elements of episodic memory, Oxford University Press.

11. Tulving, E. (1985) Memory and Consciousness. Canadian Psychology-Psychologie Canadienne 26 (1), 1-12.

12. Tulving, E. (2001) Episodic memory and common sense: how far apart? Philosophical Transactions of the Royal Society of London Series B-Biological Sciences 356 (1413), 1505-1515.

13. Tulving, E. (2002) Episodic memory: From mind to brain. Annual Review of Psychology 53, 1-25.

14. Tulving, E. (2005) Episodic Memory and Autonoesis: Uniquely Human? In The missing link in cognition: Origins of self-reflective consciousness. (Terrace, H.S. and Metcalfe, J. eds), pp. 3-56, Oxford University Press.

15. Lambon Ralph, M.A. et al. (2017) The neural and computational bases of semantic cognition. Nat Rev Neurosci 18 (1), 42-55.

16. Binder, J.R. et al. (2009) Where Is the Semantic System? A Critical Review and Meta-Analysis of 120 Functional Neuroimaging Studies. Cerebral Cortex 19 (12), 2767-2796.

17. Martin, A. (2007) The representation of object concepts in the brain. Annual Review of Psychology 58 (Journal Article), 25-45.

18. Barsalou, L.W. (2008) Grounded cognition. Annu Rev Psychol 59, 617-45.

19. Martin, A. (2016) GRAPES-Grounding representations in action, perception, and emotion systems: How object properties and categories are represented in the human brain. Psychonomic Bulletin \& Review 23 (4), 979-990.

20. Pulvermuller, F. (2013) Semantic embodiment, disembodiment or misembodiment? In search of meaning in modules and neuron circuits. Brain and Language 127 (1), 86-103.

21. Leshinskaya, A. and Caramazza, A. (2016) For a cognitive neuroscience of concepts: Moving beyond the grounding issue. Psychon Bull Rev 23 (4), 991-1001. 
22. Chen, L. and Rogers, T.T. (2014) Revisiting domain-general accounts of category specificity in mind and brain. WIREs Cognitive Science 5, 327-344.

23. Allport, D.A. (1985) Distributed memory, modular subsystems and dysphasia. In Current perspectives in dysphasia. (Edinburgh: Churchill Livingstone. edn) (Newman, S.K. and Epstein, R. eds), pp. 207-244.

24. Warrington, E.K. and Shallice, T. (1984) Category Specific Semantic Impairments. Brain 107 (SEP), 829-854.

25. Chao, L.L. et al. (1999) Attribute-based neural substrates in temporal cortex for perceiving and knowing about objects. Nat Neurosci 2 (10), 913-9.

26. Simmons, W.K. et al. (2007) A common neural substrate for perceiving and knowing about color. Neuropsychologia 45 (12), 2802-10.

27. Rugg, M.D. and Thompson-Schill, S.L. (2013) Moving Forward With fMRI Data. Perspect Psychol Sci $8(1), 84-7$.

28. Hsu, N.S. et al. (2012) Chromaticity of color perception and object color knowledge. Neuropsychologia 50 (2), 327-33.

29. Novick, J.M. et al. (2009) A case for conflict across multiple domains: memory and language impairments following damage to ventrolateral prefrontal cortex. Cogn Neuropsychol 26 (6), 527-67. 30. Badre, D. and Wagner, A.D. (2007) Left ventrolateral prefrontal cortex and the cognitive control of memory. Neuropsychologia 45 (13), 2883-901.

31. Barsalou, L.W. et al. (2018) Moving beyond the distinction between concrete and abstract concepts. Philosophical Transactions of the Royal Society B-Biological Sciences 373 (1752).

32. McClelland, J.L. and Rogers, T.T. (2003) The parallel distributed processing approach to semantic cognition. Nat Rev Neurosci 4 (4), 310-22.

33. Patterson, K. et al. (2007) Where do you know what you know? The representation of semantic knowledge in the human brain. Nat Rev Neurosci 8 (12), 976-87.

34. van den Heuvel, M.P. and Sporns, O. (2013) Network hubs in the human brain. Trends Cogn Sci 17 (12), 683-96.

35. Simmons, W.K. et al. (2010) The selectivity and functional connectivity of the anterior temporal lobes. Cereb Cortex 20 (4), 813-25.

36. Simmons, W.K. and Martin, A. (2009) The anterior temporal lobes and the functional architecture of semantic memory. J Int Neuropsychol Soc 15 (5), 645-9.

37. Coutanche, M.N. and Thompson-Schill, S.L. (2015) Creating Concepts from Converging Features in Human Cortex. Cereb Cortex 25 (9), 2584-93.

38. Binder, J.R. (2016) In defense of abstract conceptual representations. Psychon Bull Rev 23 (4), 1096-108.

39. Fernandino, L. et al. (2016) Heteromodal Cortical Areas Encode Sensory-Motor Features of Word Meaning. J Neurosci 36 (38), 9763-9.

40. Rugg, M.D. et al. (2015) Encoding and Retrieval in Episodic Memory: Insights from fMRI. In The Wiley Handbook on the Cognitive Neuroscience of Memory (Addis, D.R. et al. eds), John Wiley \& Sons, Ltd.

41. Danker, J.F. and Anderson, J.R. (2010) The ghosts of brain states past: remembering reactivates the brain regions engaged during encoding. Psychol Bull 136 (1), 87-102.

42. Kuhl, B.A. and Chun, M.M. (2014) Successful remembering elicits event-specific activity patterns in lateral parietal cortex. J Neurosci 34 (23), 8051-60.

43. Wing, E.A. et al. (2015) Reinstatement of individual past events revealed by the similarity of distributed activation patterns during encoding and retrieval. J Cogn Neurosci 27 (4), 679-91.

44. Rugg, M.D. and Vilberg, K.L. (2013) Brain networks underlying episodic memory retrieval. Curr Opin Neurobiol 23 (2), 255-60.

45. Benoit, R.G. and Schacter, D.L. (2015) Specifying the core network supporting episodic simulation and episodic memory by activation likelihood estimation. Neuropsychologia 75, 450-7. 
46. Thakral, P.P. et al. (2017) Decoding the content of recollection within the core recollection network and beyond. Cortex 91, 101-113.

47. Rugg, M.D. et al. (2003) Neural correlates of retrieval processing in the prefrontal cortex during recognition and exclusion tasks. Neuropsychologia 41 (1), 40-52.

48. Dobbins, I.G. et al. (2002) Executive control during episodic retrieval: Multiple prefrontal processes subserve source memory. Neuron 35 (5), 989-996.

49. King, D.R. et al. (2015) Recollection-Related Increases in Functional Connectivity Predict Individual Differences in Memory Accuracy. Journal of Neuroscience 35 (4), 1763-1772.

50. Westphal, A.J. et al. (2017) Episodic Memory Retrieval Benefits from a Less Modular Brain Network Organization. J Neurosci 37 (13), 3523-3531.

51. Cole, M.W. et al. (2013) Multi-task connectivity reveals flexible hubs for adaptive task control. Nat Neurosci 16 (9), 1348-55.

52. Jackson, R.L. et al. (2016) The Semantic Network at Work and Rest: Differential Connectivity of Anterior Temporal Lobe Subregions. Journal of Neuroscience 36 (5), 1490-1501.

53. Visser, M. et al. (2010) Semantic processing in the anterior temporal lobes: a meta-analysis of the functional neuroimaging literature. J Cogn Neurosci 22 (6), 1083-94.

54. Visser, M. and Lambon Ralph, M.A. (2011) Differential contributions of bilateral ventral anterior temporal lobe and left anterior superior temporal gyrus to semantic processes. J Cogn Neurosci 23 (10), 3121-31.

55. Visser, M. et al. (2012) Both the Middle Temporal Gyrus and the Ventral Anterior Temporal Area Are Crucial for Multimodal Semantic Processing: Distortion-corrected fMRI Evidence for a Double Gradient of Information Convergence in the Temporal Lobes. J Cogn Neurosci 24 (8), 1766-78.

56. Greenberg, D.L. et al. (2009) Impaired category fluency in medial temporal lobe amnesia: the role of episodic memory. The Journal of neuroscience : the official journal of the Society for Neuroscience 29 (35), 10900-10908.

57. Sheldon, S. and Moscovitch, M. (2012) The nature and time-course of medial temporal lobe contributions to semantic retrieval: An fMRI study on verbal fluency. Hippocampus 22 (6), 1451-1466. 58. Warren, D.E. and Duff, M.C. (2014) Not so fast: hippocampal amnesia slows word learning despite successful fast mapping. Hippocampus 24 (8), 920-33.

59. Shimamura, A.P. and Squire, L.R. (1987) A neuropsychological study of fact memory and source amnesia. J Exp Psychol Learn Mem Cogn 13 (3), 464-73.

60. Elward, R.L. and Vargha-Khadem, F. (2018) Semantic memory in developmental amnesia. Neuroscience Letters 680, 23-30.

61. Sharon, T. et al. (2011) Rapid neocortical acquisition of long-term arbitrary associations independent of the hippocampus. Proc Natl Acad Sci U S A 108 (3), 1146-51.

62. Cooper, E. et al. (2019) Little evidence for Fast Mapping (FM) in adults: A review and discussion. Cognitive Neuroscience 10 (4), 196-209.

63. Quiroga, R.Q. (2012) Concept cells: the building blocks of declarative memory functions. Nature Reviews Neuroscience 13 (8), 587-597.

64. Quiroga, R.Q. (2015) Concept Cells in the Human Brain. Advances in Cognitive Neurodynamics (Iv), 143-146.

65. Nosofsky, R.M. et al. (2018) Tests of an Exemplar-Memory Model of Classification Learning in a High-Dimensional Natural-Science Category Domain. Journal of Experimental Psychology-General 147 (3), 328-353.

66. Moscovitch, M. et al. (2016) Episodic Memory and Beyond: The Hippocampus and Neocortex in Transformation. Annu Rev Psychol 67, 105-34.

67. Sekeres, M.J. et al. (2018) The hippocampus and related neocortical structures in memory transformation. Neuroscience Letters 680, 39-53.

68. Moscovitch, M. (1995) Recovered consciousness: a hypothesis concerning modularity and episodic memory. J Clin Exp Neuropsychol 17 (2), 276-90. 
69. Bartlett, F.C. (1932) Remembering: A study in experimental and social psychology, Cambridge University Press.

70. Binder, J.R. and Desai, R.H. (2011) The neurobiology of semantic memory. Trends in cognitive sciences 15 (11), 527-536.

71. Graham, K.S. et al. (2002) Multiple inputs to episodic memory: Words tell another story. Neuropsychology 16 (3), 380-389.

72. Irish, M. et al. (2016) Preservation of episodic memory in semantic dementia: The importance of regions beyond the medial temporal lobes. Neuropsychologia 81, 50-60.

73. Desai, R.H. et al. (2018) The multifaceted abstract brain. Philosophical Transactions of the Royal Society B-Biological Sciences 373 (1752).

74. Miyashita, Y. (2019) Perirhinal circuits for memory processing. Nature Reviews Neuroscience.

75. Hoenig, K. and Scheef, L. (2005) Mediotemporal contributions to semantic processing: fMRI evidence from ambiguity processing during semantic context verification. Hippocampus 15 (5), 597609.

76. Bartha, L. et al. (2003) Medial temporal lobe activation during semantic language processing: fMRI findings in healthy left- and right-handers. Brain Res Cogn Brain Res 17 (2), 339-46.

77. Luo, J. and Niki, K. (2002) Role of medial temporal lobe in extensive retrieval of task-related knowledge. Hippocampus 12 (4), 487-94.

78. Robin, J. et al. (2019) Category specificity in the medial temporal lobe: A systematic review. Hippocampus 29 (4), 313-339.

79. Mullally, S.L. and Maguire, E.A. (2011) A new role for the parahippocampal cortex in representing space. J Neurosci 31 (20), 7441-9.

80. Matchin, W. et al. (2019) Same words, different structures: An fMRI investigation of argument relations and the angular gyrus. Neuropsychologia 125, 116-128.

81. Price, A.R. et al. (2015) Converging evidence for the neuroanatomic basis of combinatorial semantics in the angular gyrus. J Neurosci 35 (7), 3276-84.

82. Humphreys, G.F. and Lambon Ralph, M.A. (2017) Mapping Domain-Selective and Counterpointed Domain-General Higher Cognitive Functions in the Lateral Parietal Cortex: Evidence from fMRI Comparisons of Difficulty-Varying Semantic Versus Visuo-Spatial Tasks, and Functional Connectivity Analyses. Cereb Cortex 27 (8), 4199-4212.

83. Noonan, K.A. et al. (2013) Going beyond inferior prefrontal involvement in semantic control: evidence for the additional contribution of dorsal angular gyrus and posterior middle temporal cortex. J Cogn Neurosci 25 (11), 1824-50.

84. Ghosh, V.E. and Gilboa, A. (2014) What is a memory schema? A historical perspective on current neuroscience literature. Neuropsychologia 53, 104-14.

85. Spalding, K.N. et al. (2015) Investigating the Neural Correlates of Schemas: Ventromedial Prefrontal Cortex Is Necessary for Normal Schematic Influence on Memory. J Neurosci 35 (47), 1574651.

86. van Kesteren, M.T.R. et al. (2010) Retrieval of Associative Information Congruent with Prior Knowledge Is Related to Increased Medial Prefrontal Activity and Connectivity. Journal of Neuroscience 30 (47), 15888-15894.

87. Bontempi, B. et al. (1999) Time-dependent reorganization of brain circuitry underlying long-term memory storage. Nature 400 (6745), 671-5.

88. Nieuwenhuis, I.L. and Takashima, A. (2011) The role of the ventromedial prefrontal cortex in memory consolidation. Behav Brain Res 218 (2), 325-34.

89. Kahn, I. et al. (2004) Functional-neuroanatomic correlates of recollection: implications for models of recognition memory. J Neurosci 24 (17), 4172-80.

90. Mandzia, J.L. et al. (2004) fMRI differences in encoding and retrieval of pictures due to encoding strategy in the elderly. Hum Brain Mapp 21 (1), 1-14.

91. Dudai, Y. (2004) The neurobiology of consolidations, or, how stable is the engram? Annual Review of Psychology 55, 51-86. 
92. Dudai, Y. (2012) The Restless Engram: Consolidations Never End. Annual Review of Neuroscience, Vol 35 35, 227-247.

93. Squire, L.R. et al. (2015) Memory Consolidation. Cold Spring Harbor Perspectives in Biology 7 (8). 94. Ribot, R. (1882) Diseases of memory, Appleton, New York.

95. Corkin, S. (2013) Permanent Present Tense: The Unforgettable Life of the Amnesic Patient, H. M., New York: Basic Books.

96. Moscovitch, M. (2012) Memory before and after HM: An Impressionistic Historical Perspective. In Epilepsy and Memory (Zeman, A. et al. eds), Oxford University Press.

97. Kinsbourne, M. and Wood, F. (1975) Short-term memory processes and the amnesic syndrome. In Short-term Memory (Deutsch, D. and Deutsch, J.A. eds), pp. 258-291, New York: Academic.

98. Cermak, L.S. and O'Connor, M. (1983) The anterograde and retrograde retrieval ability of a patient with amnesia due to encephalitis. Neuropsychologia 21 (3), 213-234.

99. Cermak, L.S. (1984) The episodic semantic distinction in amnesia. In The neuropsychology of memory (Squire, L.R. and Butters, N. eds), pp. 52-62, Guilford Press.

100. McClelland, J.L. et al. (1995) Why there are Complementary Learning-Systems in the Hippocampus and Neocortex - Insights from the Successes and Failures of Connectionist Models of Learning and Memory. Psychological review 102 (3), 419-457.

101. Nadel, L. and Moscovitch, M. (1997) Memory consolidation, retrograde amnesia and the hippocampal complex. Current opinion in neurobiology 7 (2), 217-227.

102. Sekeres, M.J. et al. (2018) Changes in patterns of neural activity underlie a time-dependent transformation of memory in rats and humans. Hippocampus 28 (10), 745-764.

103. Goshen, I. et al. (2011) Dynamics of Retrieval Strategies for Remote Memories. Cell 147 (5), 11971197.

104. Winocur, G. et al. (2010) Memory formation and long-term retention in humans and animals: Convergence towards a transformation account of hippocampal-neocortical interactions. Neuropsychologia 48 (8), 2339-2356.

105. Dede, A.J.O. et al. (2016) Autobiographical memory, future imagining, and the medial temporal lobe. Proceedings of the National Academy of Sciences of the United States of America 113 (47), 13474-13479.

106. Kopelman, M.D. et al. (1999) Retrograde amnesia in patients with diencephalic, temporal lobe or frontal lesions. Neuropsychologia 37 (8), 939-958.

107. Lah, S. et al. (2006) Effects of temporal lobe epilepsy on retrograde memory. Epilepsia 47 (3), 615-625.

108. Squire, L.R. and Wixted, J.T. (2011) The cognitive neuroscience of human memory since H.M. Annu Rev Neurosci 34, 259-88.

109. Winocur, G. and Moscovitch, M. (2011) Memory transformation and systems consolidation. J Int Neuropsychol Soc 17 (5), 766-80.

110. St-Laurent, M. et al. (2014) The perceptual richness of complex memory episodes is compromised by medial temporal lobe damage. Hippocampus 24 (5), 560-576.

111. St-Laurent, M. et al. (2009) Determinants of autobiographical memory in patients with unilateral temporal lobe epilepsy or excisions. Neuropsychologia 47 (11), 2211-2221.

112. Sekeres, M.J. et al. (2016) Recovering and preventing loss of detailed memory: differential rates of forgetting for detail types in episodic memory. Learning \& Memory 23 (2), 72-82.

113. Bonasia, K. et al. (2018) Prior knowledge modulates the neural substrates of encoding and retrieving naturalistic events at short and long delays. Neurobiol Learn Mem 153 (Pt A), 26-39.

114. van Kesteren, M.T.R. et al. (2012) How schema and novelty augment memory formation. Trends in Neurosciences 35 (4), 211-219.

115. Bonnici, H.M. et al. (2012) Detecting Representations of Recent and Remote Autobiographical Memories in vmPFC and Hippocampus. Journal of Neuroscience 32 (47), 16982-16991.

116. Bonnici, H.M. and Maguire, E.A. (2018) Two years later - Revisiting autobiographical memory representations in vmPFC and hippocampus. Neuropsychologia 110, 159-169. 
117. Irish, M. (2019) On the interaction between episodic and semantic representations - constructing a unified account of imagination. In The Cambridge Handbook of Imagination (Abraham, A. ed), Cambridge University Press.

118. Cabeza, R. and Moscovitch, M. (2013) Memory Systems, Processing Modes, and Components: Functional Neuroimaging Evidence. Perspect Psychol Sci 8 (1), 49-55.

119. McCormick, C. et al. (2015) Functional and Effective Hippocampal-Neocortical Connectivity During Construction and Elaboration of Autobiographical Memory Retrieval. Cerebral Cortex 25 (5), 1297-1305.

120. de Toledo-Morrell, L. et al. (2000) Hemispheric differences in hippocampal volume predict verbal and spatial memory performance in patients with Alzheimer's disease. Hippocampus 10 (2), 136-42. 121. Irish, M. et al. (2016) Preservation of episodic memory in semantic dementia: The importance of regions beyond the medial temporal lobes. Neuropsychologia 81, 50-60.

122. Greene, J.D. et al. (1995) Autobiographical memory and executive function in early dementia of Alzheimer type. Neuropsychologia 33 (12), 1647-1670.

123. Irish, M. et al. (2011) Impaired capacity for autonoetic reliving during autobiographical event recall in mild Alzheimer's disease. Cortex 47 (2), 236-249.

124. Irish, M. et al. (2011) Profiles of recent autobiographical memory retrieval in semantic dementia, behavioural-variant frontotemporal dementia, and Alzheimer's disease. Neuropsychologia 49 (9), 2694-702.

125. Barnabe, A. et al. (2012) Autobiographical memory in mild cognitive impairment and Alzheimer's disease: A comparison between the Levine and Kopelman interview methodologies. Hippocampus 22 (9), 1809-25.

126. Strikwerda-Brown, C. et al. (2018) External details revisited - A new taxonomy for coding 'nonepisodic' content during autobiographical memory retrieval. J Neuropsychol.

127. Renoult, L. et al. (2012) Personal semantics: at the crossroads of semantic and episodic memory. Trends Cogn Sci 16 (11), 550-8.

128. Irish, M. and Piguet, O. (2013) The pivotal role of semantic memory in remembering the past and imagining the future. Front Behav Neurosci 7, 27.

129. Hodges, J.R. and Patterson, K. (2007) Semantic dementia: a unique clinicopathological syndrome. The Lancet Neurology 6 (11), 1004-1014.

130. Graham, K.S. et al. (1999) Episodic memory: new insights from the study of semantic dementia. Curr Opin Neurobiol 9 (2), 245-50.

131. Nestor, P.J. et al. (2006) Declarative memory impairments in Alzheimer's disease and semantic dementia. Neuroimage 30 (3), 1010-1020.

132. Trope, Y. and Liberman, N. (2010) Construal-Level Theory of Psychological Distance. Psychological Review 117 (2), 440-463.

133. La Corte, V. and Piolino, P. (2016) On the Role of Personal Semantic Memory and Temporal Distance in Episodic Future Thinking: The TEDIFT Model. Frontiers in Human Neuroscience 10.

134. Irish, M. et al. (2014) Grey and white matter correlates of recent and remote autobiographical memory retrieval--insights from the dementias. PLoS One 9 (11), e113081.

135. Schacter, D.L. et al. (2012) The future of memory: remembering, imagining, and the brain. Neuron 76 (4), 677-94.

136. Irish, M. and Piolino, P. (2016) Impaired capacity for prospection in the dementias - Theoretical and clinical implications. British Journal of Clinical Psychology 55 (1), 49-68.

137. Duval, C. et al. (2012) What happens to personal identity when semantic knowledge degrades? A study of the self and autobiographical memory in semantic dementia. Neuropsychologia 50 (2), 25465.

138. Irish, M. et al. (2012) Considering the role of semantic memory in episodic future thinking: evidence from semantic dementia. Brain 135 (Pt 7), 2178-91. 
139. Irish, M. (2016) Semantic memory as the essential scaffold for future oriented mental time travel. In Seeing the Future: Theoretical Perspectives on Future-Oriented Mental Time Travel (Michaelian, K. et al. eds), pp. 388-408, Oxford University Press.

140. Irish, M. et al. (2012) Exploring the content and quality of episodic future simulations in semantic dementia. Neuropsychologia 50 (14), 3488-95.

141. Strikwerda-Brown, C. et al. (submitted) Episodic and semantic interdependencies during future thinking: A fine-grained examination of 'non-episodic' content. Neuropsychologia.

142. Irish, M. et al. (2017) Damage to right medial temporal structures disrupts the capacity for scene construction-a case study. Hippocampus 27 (6), 635-641.

143. Addis, D.R. (2018) Are episodic memories special? On the sameness of remembered and imagined event simulation. Journal of the Royal Society of New Zealand, 1-25.

144. Ingvar, D.H. (1985) "Memory of the future": an essay on the temporal organization of conscious awareness. Hum Neurobiol 4 (3), 127-36.

145. Mandler, G. (2008) Familiarity Breeds Attempts: A Critical Review of Dual-Process Theories of Recognition. Perspect Psychol Sci 3 (5), 390-9.

146. Wolters, G. and Logan, G. (1998) Introduction: Fluency and remembering. Acta Psychologica 98 (2-3), 121-125.

147. Johnston, W.A. et al. (1985) Perceptual Fluency and Recognition Judgments. Journal of Experimental Psychology-Learning Memory and Cognition 11 (1), 3-11.

148. Wang, W.C. and Yonelinas, A.P. (2012) Familiarity and conceptual implicit memory: Individual differences and neural correlates. Cognitive Neuroscience 3 (3-4), 213-214.

149. Wang, W.C. and Yonelinas, A.P. (2012) Familiarity is related to conceptual implicit memory: An examination of individual differences. Psychonomic Bulletin \& Review 19 (6), 1154-1164.

150. Toth, J.P. (1996) Conceptual automaticity in recognition memory: levels-of-processing effects on familiarity. Can J Exp Psychol 50 (1), 123-38.

151. Wagner, A.D. et al. (1997) Dissociations between familiarity processes in explicit recognition and implicit perceptual memory. J Exp Psychol Learn Mem Cogn 23 (2), 305-23.

152. Yonelinas, A.P. (2002) The nature of recollection and familiarity: A review of 30 years of research. Journal of Memory and Language 46 (3), 441-517.

153. Reder, L.M. et al. (2009) Memory systems do not divide on consciousness: Reinterpreting memory in terms of activation and binding. Psychol Bull 135 (1), 23-49.

154. Henke, K. (2010) A model for memory systems based on processing modes rather than consciousness. Nat Rev Neurosci 11 (7), 523-32.

155. Berry, C.J. et al. (2012) Models of recognition, repetition priming, and fluency: exploring a new framework. Psychol Rev 119 (1), 40-79.

156. Kim, H. (2019) Neural correlates of explicit and implicit memory at encoding and retrieval: A unified framework and meta-analysis of functional neuroimaging studies. Biol Psychol.

157. Renoult, L. et al. (2015) Autobiographically significant concepts: more episodic than semantic in nature? An electrophysiological investigation of overlapping types of memory. J Cogn Neurosci 27 (1), 57-72.

158. Wimmer, G.E. and Shohamy, D. (2012) Preference by association: how memory mechanisms in the hippocampus bias decisions. Science 338 (6104), 270-3.

159. Sheldon, S. and Moscovitch, M. (2010) Recollective performance advantages for implicit memory tasks. Memory (Hove, England) 18 (7), 681-697.

160. Tulving, E. (1983) Elements of episodic memory, Clarendon Press ;

Oxford University Press.

161. Moscovitch, M. (2008) The hippocampus as a "Stupid," domain-specific module: Implications for theories of recent and remote memory, and of imagination. Canadian Journal of Experimental Psychology-Revue Canadienne De Psychologie Experimentale 62 (1), 62-79.

162. Squire, L.R. (1992) Memory and the hippocampus: a synthesis from findings with rats, monkeys, and humans. Psychol Rev 99 (2), 195-231. 
163. Tulving, E. and Schacter, D.L. (1990) Priming and Human-Memory Systems. Science 247 (4940), 301-306.

164. Neely, J.H. and Kahan, T.A. (2001) Is semantic activation automatic? A critical re-evaluation. Nature of Remembering, 69-93.

165. Rossell, S.L. et al. (2003) The anatomy and time course of semantic priming investigated by fMRI and ERPs. Neuropsychologia 41 (5), 550-64.

166. Gold, B.T. et al. (2006) Dissociation of automatic and strategic lexical-semantics: functional magnetic resonance imaging evidence for differing roles of multiple frontotemporal regions. J Neurosci 26 (24), 6523-32.

167. Lau, E.F. et al. (2013) Automatic semantic facilitation in anterior temporal cortex revealed through multimodal neuroimaging. J Neurosci 33 (43), 17174-81.

168. Sheikh, U.A. et al. (2019) Decoding the meaning of unconsciously processed words using fMRIbased MVPA. Neuroimage.

169. Suddendorf, T. and Corballis, M.C. (1997) Mental time travel and the evolution of the human mind. Genetic Social and General Psychology Monographs 123 (2), 133-167.

170. Suddendorf, T. and Corballis, M.C. (2007) The evolution of foresight: What is mental time travel, and is it unique to humans? Behavioral and Brain Sciences 30 (3), 299-+.

171. Tulving, E. (1985) Memory and Consciousness. Canadian Psychology 26, 1-12.

172. Klein, S.B. (2013) Making the case that episodic recollection is attributable to operations occurring at retrieval rather than to content stored in a dedicated subsystem of long-term memory. Front Behav Neurosci 7, 3.

173. Nigro, G. and Neisser, U. (1983) Point of View in Personal Memories. Cognitive psychology 15 (4), 467-482.

174. Robinson, J.A. and Swanson, K.L. (1993) Field and observer modes of remembering. Memory 1 (3), 169-84.

175. St Jacques, P.L.S. et al. (2017) Shifting visual perspective during retrieval shapes autobiographical memories. Neuroimage 148, 103-114.

176. Irish, M. et al. (2010) Exploring the recollective experience during autobiographical memory retrieval in amnestic mild cognitive impairment. Journal of the International Neuropsychological Society 16 (3), 546-555.

177. Tulving, E. (2005) Episodic memory and autonoesis: Uniquely human? In The Missing Link in Cognition (Terrace, H.S. and Metcalfe, J. eds), Oxford University Press.

178. Corballis, M.C. (2013) Mental time travel: a case for evolutionary continuity. Trends in Cognitive Sciences 17 (1), 5-6.

179. Cheng, S. et al. (2016) Dissociating memory traces and scenario construction in mental time travel. Neuroscience and Biobehavioral Reviews 60, 82-89.

180. Wheeler, M.A. et al. (1997) Toward a theory of episodic memory: the frontal lobes and autonoetic consciousness. Psychol Bull 121 (3), 331-54.

181. Nyberg, L. et al. (2010) Consciousness of subjective time in the brain. Proc Natl Acad Sci U S A 107 (51), 22356-9.

182. Miloyan, B. et al. (2019) Measuring mental time travel: Is the hippocampus really critical for episodic memory and episodic foresight? Cortex.

183. Ekstrom, A.D. et al. (2018) Human Spatial Navigation, Princeton University Press.

184. Eichenbaum, H. and Cohen, N.J. (2014) Can we reconcile the declarative memory and spatial navigation views on hippocampal function? Neuron 83 (4), 764-70.

185. Herdman, K.A. et al. (2015) Impoverished descriptions of familiar routes in three cases of hippocampal/medial temporal lobe amnesia. Cortex 71, 248-63.

186. Hassabis, D. et al. (2007) Patients with hippocampal amnesia cannot imagine new experiences. Proceedings of the National Academy of Sciences of the United States of America 104 (5), 1726-1731. 187. Mullally, S.L. et al. (2014) Scene construction in developmental amnesia: an fMRI study. Neuropsychologia 52, 1-10. 
188. Tu, S. et al. (2015) Lost in spatial translation - A novel tool to objectively assess spatial disorientation in Alzheimer's disease and frontotemporal dementia. Cortex 67, 83-94.

189. Rubin, D.C. and Umanath, S. (2015) Event memory: A theory of memory for laboratory, autobiographical, and fictional events. Psychol Rev 122 (1), 1-23.

190. Rubin, D.C. et al. (2019) Scenes enable a sense of reliving: Implications for autobiographical memory. Cognition 183, 44-56.

191. Hassabis, D. and Maguire, E.A. (2007) Deconstructing episodic memory with construction. Trends in cognitive sciences 11 (7), 299-306.

192. Maguire, E.A. and Mullally, S.L. (2013) The hippocampus: a manifesto for change. J Exp Psychol Gen 142 (4), 1180-9.

193. Ryan, L. et al. (2008) Hippocampal activation during episodic and semantic memory retrieval: comparing category production and category cued recall. Neuropsychologia 46 (8), 2109-2121.

194. Hoscheidt, S.M. et al. (2010) Hippocampal activation during retrieval of spatial context from episodic and semantic memory. Behav Brain Res 212 (2), 121-32.

195. de Chastelaine, M. et al. (2016) The neural correlates of recollection and retrieval monitoring: Relationships with age and recollection performance. Neuroimage 138, 164-175.

196. Thakral, P.P. et al. (2015) Cortical reinstatement and the confidence and accuracy of source memory. Neuroimage 109, 118-129.

\section{Figure legends}

\section{Figure 1:}

This figure illustrates the close overlap between the general semantic network $(A)$ and the episodic core recollection network (B). The two networks share essentially the same parahippocampal, middle temporal, ventral parietal and midline frontal and posterior regions. In contrast, the hippocampus is present in the core recollection network (B) but not in its semantic counterpart ( $\mathrm{A}$; see main text for discussion). Although, the anterior temporal lobe $(A T L)$ is absent from both depicted networks, (likely due to the degradation of signal quality in this region), $\mathrm{fMRI}$ studies using sequences optimized to detect BOLD signals from ATL have reported that increased activity in this brain region during conceptual processing, especially in anterior fusiform gyrus $[53,54]$. Although it has yet to be established whether the ATL consistently demonstrates equivalent episodic retrieval effects with optimized fMRI, figure $1 \mathrm{C}$, depicting the outcome of the contrast between accurate and inaccurate associative 
recognition memory judgments on word pairs, suggests that this is a distinct possibility. A: The general semantic network as revealed by $\mathrm{fMRI}$ meta-analysis [38]. DMPFC = dorsomedial prefrontal cortex; FG/PH = fusiform gyrus/parahippocampal cortex; IFG = inferior frontal gyrus; IPC = inferior parietal cortex; $P C=$ posterior cingulate/precuneus; VMPFC $=$ ventromedial prefrontal cortex. Adapted with permission from [38]. B: The core recollection network [44]. Adapted with permission from [44]. C: Recollection-related enhancement of $\mathrm{FMRI} B O L D$ signal in bilateral anterior ventral temporal cortex (thresholded at $p<.001$, with family-wise error correction $(p<.05)$ at the cluster level). The figure depicts the outcome of the contrast between accurate and inaccurate associative recognition memory judgments on word pairs, a contrast assumed to permit identification of neural correlates of successful recollection. These findings were originally reported in [195], but did not include a depiction of the ventral temporal effects illustrated here. The data were obtained from a sample of 136 participants ranging in age from 18-76 yrs. For further details see [195].

\section{Figure 2:}

This figure displays two examples of episodic reinstatement effects (visual: left and auditory: right), when cortical activity elicited as a recollected event was initially experienced is reactivated during successful recollection [196]. Subjects studied pictures in association with their visually or auditorily presented names. At test, they discriminated studied pictures according to the modality of the associated name. Yellow clusters indicate where modality-

selective source recollection effects (high>low confidence judgments) overlapped with 
modality effects at study (visual>auditory and vice-versa). Adapted with permission from [196]. 